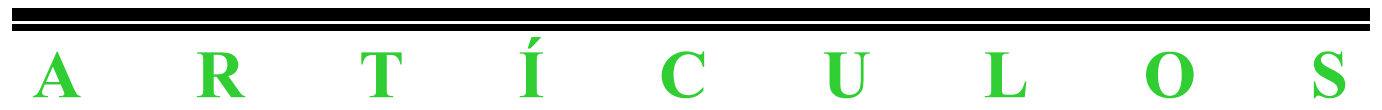




\section{LA NOCIÓN DE PROCEDIMIENTO EN LA TEORÍA DE LA ARGUMENTACIÓN JURÍDICA}

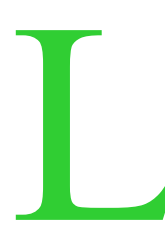

a noción de procedimiento es una noción clave en las teorías actuales de la argumentación jurídica y de la argumentación práctica racional. Algunos autores hablan incluso de una «teoría procesal de la argumentación»: de acuerdo con tal teoría, una norma es justa (richtig) si es el resultado de un determinado procedimiento, el del discurso práctico racional ${ }^{1}$. La teoría tiene diversos presupuestos e implicaciones: algunos de ellos son filosóficamente triviales, ya reconocidos y ampliamente difundidos en la literatura filosófica; otros son más exigentes, controvertibles y controvertidos.

Es inherente a nuestra cultura filosófica y jurídica la idea misma de que la racionalidad es un procedimiento, o un método; que ser racional no significa nada más que actuar de una manera racional. Muchas razones pequeñas desplazan a la «Razón» mayúscula. El procedimiento racional es una carrera de obstáculos, en la que aportar una razón significa superar un obstáculo, dar un paso adelante hacia la conclusión, al reducir la serie de conclusiones argumentativamente permitidas (si, al final, podemos tener sólo una conclusión, o no, es asunto altamente controvertible).

Estos caracteres identifican la caracterización más débil de la teoría procesal de la racionalidad. Esta no es algo peculiar de la razón práctica: el concepto kantiano de razón formal contiene ya, de hecho, este concepto de racionalidad procesal, de la que derivan las pretensiones de validez de nuestras creencias justificadas, no sólo

${ }^{1}$ R. Alexy, «Die Idee einer prozeduralen Theorie der juristischen Argumentation», en Rechtstheorie, Beiheft 2: «Methodologie und Erkenntnistheorie der juristischen Argumentation», pág. 176. 
en la esfera práctico-moral, sino también en las esferas teorética y estética².

2. Otras dos teorías del procedimiento son, definitivamente, mucho más exigentes. Ambas han sido construidas para resolver problemas práctico-morales; cada una de ellas se fundamenta en una concepción específica de las relaciones entre racionalidad científica y racionalidad práctica. Mi tesis es que los dos grupos de reglas distinguidos por Alexy en su código de la razón práctica ${ }^{3}$, se refieren, cada una de ellas, a estas dos diferentes concepciones del procedimiento; es decir, que no son reglas procedimentales en el mismo sentido de la expresión.

De acuerdo con la primera de ellas, una (menos) débil concepción de la racionalidad procedimental, la que llamaré teoría de la racionalidad de-las-reglas-del-juego, el procedimiento no es más que un sustitutivo, aunque sea precioso o verdaderamente inevitable. Necesitamos un procedimiento como un conjunto de reglas-del-juego siempre que hemos de alcanzar un consenso sobre materias que no pueden ser juzgadas en términos de verdad o de justicia material. De acuerdo con esta teoría, el procedimiento identifica una clase de racionalidad formal, la única que rige propiamente en el campo de las normas, de los debe, de los valores, de las ideologías.

De acuerdo con esta concepción (soberbiamente representada por la concepción de la democracia de Kelsen ${ }^{4}$ ) una conclusión que se obtenga siguiendo las reglas no puede definirse como correcta o como justa por esa misma razón. De acuerdo con esta concepción, la justicia, concebida como un valor intrínseco y no derivado, como un valor último y no instrumental, no es demostrable. No hay prueba para la justicia: no la hay de racionalidad, ni tampoco de racionalidad procedimental.

Los valores, y entre ellos el valor de la justicia, ciertamente existen; pero son irracionales, o mejor no-racionales. Afirmar o aceptar un valor es un acto monológico, no relacionado con reglas: por consiguiente, nada tiene que ver con la racionalidad. Las reglas y los procedimientos son necesarios cuando no se trata de asumir un valor, sino de imponerlo: siempre que se trata de dictar normas. En este preciso momento, de acuerdo con esta concepción, las reglas

${ }^{2}$ Ver J. Habermas, Moralbewusstsein und kommunikatives Handeln, Frankfurt a. M., Suhrkamp, 1983, cap. 1 .

${ }^{3} \mathrm{R}$. Alexy, Theorie der juristischen Argumentation. Die Theorie des rationalen Diskurses als Theorie der juristischen Begtründung, Frankfurt a. M., Suhrkamp, 1978, B. 4.

${ }^{4}$ Ver H. Kelsen, Vom Wesen und Wert der Demokratie, Tübinge, Mohr, 1929. 
no sólo han de existir: también han de ser racionales. Racionales en el sentido de racionalidad práctica, que sólo puede querer decir racionalidad instrumental, idoneidad para el fin. El fin de que se trata es el de acercar tanto como sea posible la utopía de una libertad absoluta, de una ausencia de restricciones y de deberes, donde no existan normas dictadas, sino sólo valores ampliamente difundidos, universalmente aceptados.

El modelo al que esta concepción hace referencia esencialmente es la clase de procedimiento que se ejemplifica en las reglas de la mayoría de la democracia parlamentaria. Esta se concibe como un procedimiento racional, más que como una racionalidad procedimental; y se distingue del procedimiento científico esencialmente porque sus reglas no tratan de justificar el contenido del juicio que se obtiene siguiendo el procedimiento; lo que se pretende es que las reglas coloquen a este juicio de antemano a resguardo de los ataques discrepantes. El resultado del procedimiento, entonces, no es visto como la conclusión de un procedimiento, sino que realmente sigue siendo el resultado de una decisión; esta decisión, ciertamente, se ha alcanzado siguiendo las reglas, pero no hay una relación material entre las reglas aplicadas y la decisión.

La regla fundamental, de acuerdo con esta concepción del procedimiento, es el principio de la mayoría. Es correcto y racional seguir este principio; lo es, sin embargo, no porque la resolución mayoritaria sea la resolución correcta y racional. De hecho, la observancia de las reglas procedimentales, al dejar por completo intocado el asunto de que se trate, producen una restricción meramente externa. Internamente, cada cual es totalmente libre de ver como justa, o como más justa, otra resolución que pudiera haberse adoptado.

Esta concepción del procedimiento, entonces, tiene un componente que es una característica peculiar de la modernidad: me refiero a la distinción entre la actitud externa y la creencia interna, entre el Derecho y la moral. El imperio de la práctica, de la creencia, de la moralidad es el imperio de la libertad; dentro de él la conciencia individual tiene sus propias certezas, aunque las mismas no son demostrables.

3. En este sentido, la tercera concepción de la racionalidad procedimental -la concepción fuerte, de la que voy a hablar- no es «moderna»: su meta es la restitución de la racionalidad a los asuntos práctico-morales.

Exactamente de la misma forma que una proposición descriptiva, una proposición normativa -de acuerdo con esta teoría- pretende y aspira a la racionalidad, lo que equivale a estar racionalmente 
justificada. No es lo mismo decir: «Debes hacer esto y aquello» que decir «iHaz esto y aquello!». Si digo «¡Haz esto y aquello!» o «¡Ven aquí!», «¡Páralo!», «¡Cállate!», no hay algo en juego más que relaciones entre tú y yo. Estoy sólo desafiándote a que confrontemos nuestras fuerzas: y puedo, bien tener éxito, bien fracasar. Mi oración imperativa, entonces, no es un discurso; es una acción estratégica ${ }^{5}$. La situación es totalmente diferente si digo «Debes hacer esto y aquello», o «debes venir aquí», «debes pararlo», o cualquier otra cosa. Haciendo esto, estoy o bien ejerciendo autoridad o bien simplemente dándote un fragmento de información. En el primer caso, estoy haciendo algo más que ejercer poder; en el segundo, estoy haciendo otra cosa.

Al decir «debes», estoy afirmando implícitamente que estoy preparado para implicarme en un discurso o acción comunicativa. Al decir «debes» presupongo como permisible, en principio, una confrontación acerca de las razones por las cuales debes.

La confrontación acerca de las razones en favor de una proposición normativa cuenta como un discurso o una argumentación práctica racional. No tiene, qua premisas, principios generales a aplicar: tiene, qua premisas, proposiciones normativas que han de ser justificadas, en la medida en que se requiera tal justificación.

Qué razón, o qué clase de razones pueden contar como una buena razón, es decir, como una justificación en favor de una proposición normativa es enjuiciado por un conjunto de reglas que articulan, conjuntamente, el procedimiento de la argumentación práctica racional. El resultado del procedimiento es una proposición normativa justa, o justificada.

4. Me parece que las diferencias entre esta teoría procedimental fuerte y la teoría moderadamente débil -la teoría a la que he llamado la teoría de la racionalidad de las-reglas-del juego son muy claras.

En primer lugar, en la teoría procedimental fuerte, el procedimiento no es visto como un mero sustituto. No se trata de que allí donde, como en la esfera práctico-moral, la objetividad sea inalcanzable uno haya de contentarse con el consenso. De lo que se trata es de que objetividad significa consenso.

Esta idea puede desarrollarse de diferentes maneras. La versión más fuerte es la de Habermas, autor de una teoría consensual de la verdad. Pero ahora, dejando aparte el perplejante asunto de la verdad, limitémonos a reflexionar acerca de cómo la identificación entre objetividad y consenso restaura la conexión entre racionalidad

${ }^{5}$ J. Habermas, Theorie des kommunikativen Handelns, Frankfurt a. M., Suhrkamp, 1981. formal 
material y racionalidad formal que la teoría de la racionalidad de las-reglas-del-juego había roto (y restaura también, al mismo tiempo, la conexión entre justicia material y justicia formal).

En este sentido de «procedimiento», la distancia entre el procedimiento racional en asuntos práctico-morales y el procedimiento racional en el razonamiento científico resulta acortada. En ambas situaciones, si alguien no tiene nada que objetar a la corrección del uso del método, o bien aprueba la conclusión a la que se ha llegado por medio de él, o bien tiene algo que objetar (quizá en términos de oportunidad) a la selección del método. Lo que no puede hacer consistentemente es estar conforme con, pero no convencido por, el resultado del procedimiento. En el imperio de la racionalidad, sea teorética o práctica, si el procedimiento se ha desarrollado correctamente, el resultado es correcto. En lo que concierne a la racionalidad práctica, de acuerdo con esta concepción, el procedimiento racional no es más que el lugar en el que los valores son puestos a prueba.

El modelo al que esta concepción hace referencia esencialmente es la clase de procedimiento diseñado por las reglas de los procesos jurídicos, de los procedimientos judiciales. Lo peculiar de esta clase de procedimiento es que en él, en efecto (a diferencia del procedimiento parlamentario), se alegan pruebas. Al final del procedimiento, se adopta aquella decisión que, de acuerdo con las reglas, ha sido descubierta -es decir, probadacomo correcta. La voluntad del juez, como componente de la decisión, es mucho menos importante que su certidumbre. Ambas son componentes internos, pero mientras que la voluntad es por definición arbitraria, la certidumbre -por definición- ha de estar fundamentada. Ahora bien, tenemos una antigua concepción del procedimiento judicial, de acuerdo con la cual la decisión del juez está fundamentada en reglas, y, por consiguiente, cuando el juez tiene certidumbre, tiene responsabilidad también. La concepción moderna es diferente: de acuerdo con ella, la tarea del juez es aplicar reglas con exactitud, más que alcanzar certidumbre; como consecuencia, se supone que el responsable de la particular decisión obtenida es el autor de las reglas y no el juez.

Resulta claro que aquí estamos frente a dos diferentes significados de la frase «fundamentado en reglas». Vale la pena hacer notar que encontramos la misma diferencia de interpretación del procedimiento judicial en una controversia entre los dos principales elaboradores de la teoría procedimental de la racionalidad práctica. Jürgen Habermas, por un lado, no concibe el procedimiento judicial como un discurso, sino como una acción estratégica llevada a cabo por dos partes enfrentadas; en su opinión, las partes no pueden pretender un fin común, porque su objetivo no es obtener la verdad, 
sino decidir un caso, y cada parte pretende una decisión favorable ${ }^{6}$. Robert Alexy objeta: «Las partes o sus abogados plantean con sus intervenciones una pretensión de corrección, aunque sólo persigan intereses subjetivos. Lo que exponen como razones en favor de una determinada decisión podría, al menos en principio, estar incluido en un tratado científico-jurídico. No es infrecuente, sobre todo en procesos ante altos tribunales, que las fundamentaciones procedan de discusiones doctrinales. Además, los argumentos formulados ante el tribunal son recogidos a menudo en las fundamentaciones judiciales y difícilmente puede ponerse en duda que éstas se elaboran bajo la pretensión de corrección» ${ }^{7}$.

5. Puede ser que los dos autores no se refieran a la misma cosa: puede ser que Habermas tenga en mente los comportamientos reales, mientras que Alexy tendría en mente modelos ideales. Pero, si no tomamos en cuenta el nivel fenomenológico, podemos también decir que Jürgen Habermas presupone como un modelo del procedimiento judicial moderno el procedimiento judicial que Alessandro Giuliani ha llamado «asimétrico»; mientras que Robert Alexy, al concebir los procedimientos judiciales como discursos, adopta un paradigma pre-moderno, el procedimiento judicial que Alessandro Giuliani ha llamado «isonómico» ${ }^{8}$.

Pues bien: estoy segura de que hay una relación estrecha entre el modelo del discurso práctico racional y el del procedimiento judicial isonómico, pero en mi opinión la relación no es tal como la presenta Alexy. Encuentro más aceptable un tipo de relación como la presentada por Perelman, de acuerdo con la cual las figuras de los participantes en la argumentación práctica racional están modeladas sobre la del juez en el procedimiento judicial isonómico, y no viceversa9 ${ }^{9}$.

Junto con esto, deseo subrayar que una de las principales fuentes de la teoría procedimental de la argumentación es la teoría de la retórica, a la que hace referencia el propio paradigma isonómico del procedimiento judicial.

${ }^{6}$ J. Habermas, «Theorie der Gesellschaft oder Sozialtechnologie?», en J. Habermas/N. Luhmann, Theorie der Gesellschaft oder Sozialtechnologie?, Frankfurt a. M., Suhrkamp, 1971, págs. 149-290, en pág. 200.

${ }^{7}$ R. Alexy, Theorie der juristischen Argumentation, cit., pág. 270.

8 A. Giuliani, «Ordine isonomico ed ordine asimmetrico: nuova retorica e teoria del processo», Sociologia del diritto, 1986, págs. 81-90.

${ }^{9}$ Ver Ch. Perelman, «Ce que la philosophie peut apprendre par l'étude du droit», en Ch. Perelman, Droit, moral et philosophie, París, Pichon et Durand-Auzias, 1968, págs.135-147. 
La regla fundamental del procedimiento, de acuerdo con esta concepción, es el principio de universalización. Este principio es claramente, por ejemplo, la matriz de algunas reglas muy importantes en el código de razón práctica desarrollado por Robert Alexy.

Véanse, por ejemplo, las siguientes reglas:

1.3. Todo hablante que aplique un predicado $\mathrm{F}$ a un objeto $a$ debe estar dispuesto a aplicar F también a cualquier otro objeto que sea como $a$ en todos los aspectos relevantes ${ }^{10}$.

1.3'. Todo hablante sólo puede afirmar aquellos juicios de valor o de deber que afirmaría, asimismo, en los mismos términos en todos los casos que se asemejen al caso dado en todos los aspectos relevantes ${ }^{11}$.

3.1. Quien propone tratar a una persona A de forma diferente que a una persona B está obligado a proporcionar una justificación para hacerlo así ${ }^{12}$.

Podemos, ahora, reflexionar sobre las diferencias que hay entre aplicar este principio, aplicar el principio de mayoría y aplicar el Derecho sustantivo. Me parece obvio que en primer caso de aplicación-de-regla, a diferencia del segundo, las reglas a aplicar afectan al contenido del resultado del procedimiento (al que podría llamarse, por esta misma razón, una conclusión del procedimiento mismo, y no sólo una decisión a la que se llega a través del procedimiento). Además, este primer caso, a diferencia del tercero, es una aplicación de reglas formales, las cuales no pueden más que dejar a la persona que las aplica la completa responsabilidad por el particular juicio al que llegue, es decir, por el valor sustantivo encarnado en tal juicio.

6. Ahora bien, en relación con esta versión fuerte de la teoría procedimental de la racionalidad, surge un problema espinoso, dado que esta teoría adscribe este doble carácter -son a la vez reglas formales y sustantivas- a una serie de reglas que no pueden considerarse, en ningún sentido significativo, como reglas lógicas, por cuanto son reglas acerca de la conducta de los hablantes, o reglas acerca de la admisión en los discursos.

Podemos tomar en consideración aquí la regla de razón fundamental, de acuerdo con Robert Alexy:

${ }^{10}$ R. Alexy, Theorie der juristischen Argumentation, cit., págs. 234-235.

${ }^{11}$ R. Alexy, Theorie der juristischen Argumentation, cit., pág. 237.

${ }^{12}$ R. Alexy, Theorie der juristischen Argumentation, cit., pág. 243. 
2. 1. Todo el que pueda hablar puede tomar parte en el discurso ${ }^{13}$.

¿Qué puede querer decir que una proposición normativa no está fundamentada y, en consecuencia, no puede ser justa, si alguien, contra su voluntad, fue dejado fuera del discurso? Pienso que la única manera de justificar esta regla es afirmar que la justicia, a diferencia de la verdad, no es un valor objetivo, sino, en la mejor hipótesis, un valor ampliamente difundido.

Así pues, pienso que al menos algunas de las reglas de la argumentación práctica racional no pueden ser, incluso en esta versión fuerte, más que «reglas de un juego»; lo mismo vale para el procedimiento judicial, de acuerdo con el modelo isonómico que puede considerarse como un paradigma para la teoría de la argumentación práctica racional. El fallo correcto o justo, de acuerdo con este modelo, no es ni el fallo que «se sigue lógicamente» de los mandatos dictados por una remota autoridad, ni tampoco el fallo «arbitrariamente» dictado por la autoridad efectiva que toma la decisión. El fallo correcto o justo, de acuerdo con este modelo, es el resultado de una ponderación comparativa de los argumentos, interpretaciones, pruebas alegados por las partes.

Si esta concepción del fallo justo o correcto se toma como una regla semántica de acuerdo con la cual «justo», en la medida en que se refiere a un fallo, significa: «el resultado de una ponderación comparativa de los argumentos, las interpretaciones y las pruebas alegados por las partes», yo encuentro algo difícil aceptarla.

Por otra parte, yo encuentro mucho más fácil aceptar esta concepción, si se la entiende como un principio moral, que no se refiere al significado de «justicia», sino a la selección de un método para alcanzar la decisión o conclusión justa.

Y, desgraciadamente, puede suceder que los tribunales lleguen por el procedimiento correcto a una conclusión injusta, o viceversa.

Lo mismo vale para la argumentación práctica general (en una situación real -lo séy no en la situación lingüística ideal).

Se puede obtener la conclusión de que los Negros y los Blancos, los Judíos y los Cristianos son iguales y han de ser tratados por igual en un discurso del que los Negros y los Judíos hayan sido excluidos; o se puede obtener la conclusión de que los hombres y las mujeres no deben ser tratados por igual en un discurso en el que participen hombres y mujeres.

Es probable -estoy de acuerdo- que ambas situaciones sean transitorias. Si los valores en conflicto, y en juego, son igualdad y

${ }^{13}$ R. Alexy, Theorie der juristischen Argumentation, cit., pág. 240. 
desigualdad, como en nuestro ejemplo, es casi seguro que, una vez alcanzada la conclusión de que los Católicos y los Judíos, los Negros y los Blancos son iguales, se aceptará a los Judíos y a los Negros en el discurso. Por otra parte, una vez aceptadas las mujeres como participantes en el discurso, al final se obtendrá la conclusión de que los hombres y las mujeres son iguales y han de ser tratados por igual.

¿Pero qué ocurre, por ejemplo, con la uniformidad [sameness] y la diferencia? Si los valores en conflicto, y en juego, fueran uniformidad y diferencia, yo no podría decir de antemano qué tipo de conclusión se alcanzaría en un discurso que tuviera una determinada participación y no otra ${ }^{14}$.

En la medida en que se trata de la vida real, sería mejor -pienso- distinguir entre verdad y justicia, por una parte, y el valor moral de la selección de un método para alcanzar la verdad y la justicia por otra.

Y el Derecho positivo y los procesos jurídicos son, indudablemente, asuntos de la vida real.

(Trad. de Juan Ruiz Manero)

${ }^{14}$ Ver I. M. Young, Justice and the Politics of Difference, Princeton N.J., Princeton University Press, 1990. 
DOXA-14 (1993)

$\triangle$ 